\title{
Hand Therapy for Index Flexors Underwent Repair: A Case Study
}

\section{Moussa Abu Mostafa*}

Head of Occupational Therapy Department, Hamad Hospital, Doha, Qatar

*Corresponding Author: Moussa Abu Mostafa, Head of Occupational Therapy

Department, Hamad Hospital, Doha, Qatar.
Received: February 19, 2020

Published: March 14, 2020

(C) All rights are reserved by Moussa Abu

Mostafa.

\begin{abstract}
Background: Proper surgery and hand rehabilitation protocols are fundamentals for effective hand flexor tendon repair management. Hand surgery and hand therapy literature involve many scientific papers discussing the Kessler technique, Kleinert and Duran approaches, and others. Current evidence supports golden outcomes of tendon repair that are free tendon gliding and normal hand functions. This paper presents a case of tendon repair who had a four-week hand therapy course.

Case Presentation: Our patient (A N) is a 32 years old male who used to work in construction and steel. He had left index flexor tendons Zone II laceration injury in last December 2019. A N underwent tendons' repair by the modified Kessler technique and later he was referred to the occupational therapy department for evaluation and hand therapy. His course of management included active and passive motion approach where it went smoothly and the patient showed recovery or good progress in tendon gliding, motion, function, and productive work.

Conclusions: The combination of surgical techniques and proper rehabilitation approaches bettered the results of flexor tendon repair. Early mobilization is fundamental for better tendon gliding hand functions if tendon rupture prevented.
\end{abstract}

Keywords: Hand Therapy; Occupational Therapy; Hand Flexor Tendon Injury; And Hand Flexor Tendon Repair

\section{Introduction}

There is no accurate global statistics about the hand tendon injury and repair. However, an estimated 300,000 tendon and ligament repair surgeries are performed annually in the U.S [1]. Because of hypocellular and hypovascular nature of the tendons, the natural healing process of tendons is slow and surgery is fundamental to bring both ends of the tendon together and allow better outcomes. However, this type of management involves the risk of adhesions and tendon rupture [2]. Even after one year of surgery, the structure and function of the resulting tissue remain inferior to uninjured tendons. During that, tendon adhesions, joint stiffness, tendon rupture, infection, poor tendon gliding, regional pain syndrome, and abnormal scars may occur as complications after surgery and during the rehabilitation phase. The overall rate of complications ranges from 11 - 13\% [1,3].

Hand flexors tendon injuries usually occur due to laceration from knives, glass, or sharp machines like in this case study where the injury occurred by grinder disc during construction and steelwork. Injuries by grinder disc are preventable; however, they are common injuries among construction and steelworkers. Tendon healing involves an inflammatory stage (48 - 72 hours), proliferation stage ( 1 - 4 weeks), and remodelling stage ( 6 weeks). Flexor tendon repairs are currently mobilised in dorsal splints with no active grasping with the fingers for 4 - 5 weeks. There follows a period of 3 - 4 weeks of gradual increase of activity with the splint only 
being worn for safety issues at night and in public places, where the fingers might be accidentally pulled into extension. Full use of the hand for light activities and therapy to correct failures of finger extension begins only after 8 weeks, with heavy grasping activities being avoided for 12 weeks. Patients return to sedentary manual activities at 8 -10 weeks and to heavy manual labour at 12 weeks after surgery [2].

Hand flexors tendon injuries are challenging injuries. They are close to the skin and affect a vital part of the hand functions that is grasp and pinch. Postoperative hand therapy has an extensive debate about using active and passive mobilization. Active mobilization achieves function and gliding of the tendon if the risk of tendon rupture is avoided [2]. This case study presents a patient with hand tendon flexor repair who was referred to the outpatient clinic of occupational therapy for evaluation and management.

\section{Methods}

The author in this study selected and followed up one case of a repaired hand tendon flexor injury with the aim to study in-depth the outcomes of four-week hand therapy intervention.

\section{Objectives}

1. Reporting four-week hand therapy management for index flexors underwent repair.

2. Anticipating progress; and.

3. Reflection on relevant literature.

A case study design that investigates treatment in-depth was used to frame this study. One patient who underwent index repair surgery was followed for 4 weeks in the hand therapy unit within the occupational therapy department of Hamad Hospital in the Gaza Strip in January-February 2020. Three intakes took place: admission, two-week follow-up, and four-week follow-up. Outcome tools included Oedema assessment, Rang of Motion, Sensation, Hand dynamometry, Pain, Hand function, and Productivity (ability to work).

\section{Case presentation}

A N is a 32 years old male patient from Gaza. He is right-handed and used to work in construction and steel for the last 14 years. A $\mathrm{N}$ used to use the hammer and the grinder in his work. He usually checks the grinder disc for safety every day. On the day of his work accident (06.12.2019), A N used a worn grinder disc to cut the wood where the grinder produced a strong shaking force. In a few seconds, A N lost control over the grinder and had his hand injury.
He had left index flexor tendons Zone II laceration injury. A N underwent tendons' repair by the modified Kessler technique after injury by three days in Shifa Hospital. The wrist was immobilized in a cast (dorsal slap) in 20 degrees of flexion and metacarpophalangeal joint at 40 degrees of flexion. After 6 weeks the cast was removed and hand therapy started in the occupational therapy outpatient clinic of Hamad Rehabilitation Hospital in January 2020 (Figure 1).

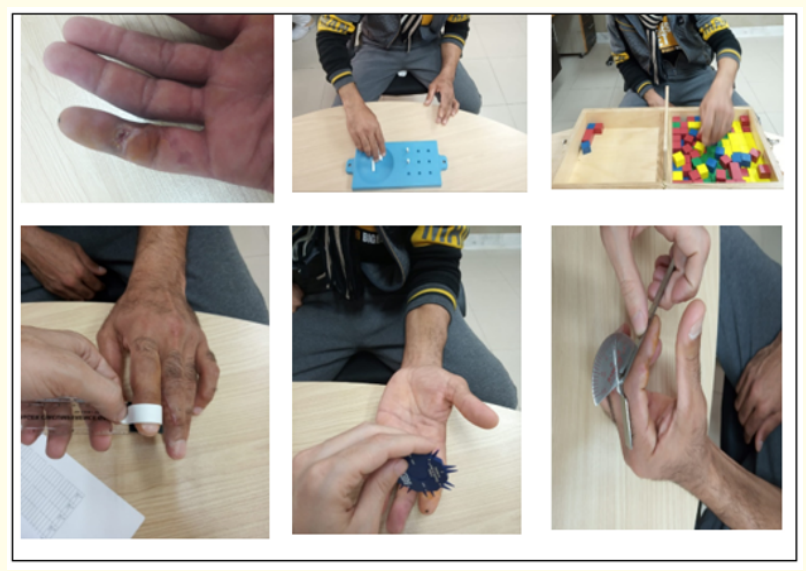

Figure 1: Illustrative photos of hand therapy assessment.

Left hand (injured) was evaluated for oedema, pain, Range of Motion (ROM), muscle power, sensation, functions, and work ability. Similarly, right hand (sound) was evaluated to set the norms for this patient and to allow comparison between both hands. Early motion protocols such as Kleinert, Duran, and others [3] were followed where passive and active motion without rubber band was enhanced within pain tolerance and normal range of motion.

Hand therapy included the following modalities:

- Patient education about wound care and prevention of complications due to sensation loss

- $\quad$ Range of Motion exercise

- Rolyan sensory re-education

- $\quad$ Bandaging

- Therapeutic putty

- Therapand exercise

- Mechanical hand tool

- Hand exercise board with hook and loop fasteners

- $\quad$ Training on the use of hammer $1 \mathrm{Kg}$ for 5 - 10 minutes. 
The plan of treatment went smoothly except for one event where the patient developed burn in his injured finger, when he unconsciously touched a hot object. At this time he was in the second week of hand therapy. Follow-up will take place after one and two months of discharge.

\section{Discussion}

Our patient in this case study had several complications after tendon laceration and repair. However, after four-week hand therapy, he showed progress in all targeted aspects of treatment.
His index oedema and pain resolved completely. His range of motion improved significantly except DIP joint passive flexion (-25 degrees) and active flexion ( -70 degrees). Sensation improved as well both two-point discrimination, pin pain, hot, and cold. The patient regained his power in the left hand both grip (36 Kgs) and pinch (7.5 Kgs). His left hand functions improved significantly: Nine-Hole Big test (18 seconds), Box and Blocks test (38 pcs/minute). He became able to hold one Kg hammer and perform ten knocks (Table 1).

\begin{tabular}{|c|c|c|c|c|c|c|c|}
\hline Name: A N & Age: 34 yrs & & & & & & \\
\hline Gender: male & $\begin{array}{l}\text { Occupation: construction- } \\
\text { andsteelworker }\end{array}$ & \multicolumn{2}{|c|}{20.01 .2020} & \multicolumn{2}{|c|}{03.02 .2020} & \multicolumn{2}{|c|}{ 17.02.2020 } \\
\hline $\begin{array}{l}\text { Admission: } \\
20.01 .2020\end{array}$ & Discharge: 20.02 .2020 & \multicolumn{2}{|c|}{ Admission } & \multicolumn{2}{|c|}{$\begin{array}{l}\text { Two weeks follow- } \\
\text { up }\end{array}$} & \multicolumn{2}{|c|}{$\begin{array}{l}\text { Four weeks follow- } \\
\text { up }\end{array}$} \\
\hline Dominant hand: Lt & & Right & Left & Right & Left & Right & Left \\
\hline \multirow[t]{2}{*}{ Edema } & MP circumference $(\mathrm{cm})$ & 3.5 & 4 & 3.5 & 3.5 & 3.5 & 3.5 \\
\hline & PP circumference $(\mathrm{cm})$ & 4 & 6 & 4 & 4 & 4 & 4 \\
\hline \multirow{2}{*}{$\begin{array}{l}\text { Passive Range of } \\
\text { Motion }\end{array}$} & MIP flexion & 90 & 0 & 90 & 90 & 90 & 90 \\
\hline & DIP flexion & 90 & 0 & 90 & 55 & 90 & 65 \\
\hline \multirow{2}{*}{$\begin{array}{l}\text { Active Range of Mo- } \\
\text { tion }\end{array}$} & MIP flexion & 90 & 55 & 90 & 70 & 90 & 85 \\
\hline & DIP flexion & 90 & 0 & 90 & 0 & 90 & 20 \\
\hline \multirow[t]{4}{*}{ Sensation } & 2-PD & $2 \mathrm{~mm}$ & Lost & $2 \mathrm{~mm}$ & $8 \mathrm{~mm}$ & $2 \mathrm{~mm}$ & $8 \mathrm{~mm}$ \\
\hline & Pin pain & Normal & Lost & Normal & Normal & Normal & Normal \\
\hline & Hot & Normal & Lost & Normal & Normal & Normal & Normal \\
\hline & Cold & Normal & Lost & Normal & Normal & Normal & Normal \\
\hline \multirow[t]{2}{*}{ Hand dynamometry } & Grip Kgs & 35 & NT & 50 & 36 & 50 & 36 \\
\hline & Pinch Kgs & 6.5 & NT & 6.5 & 6.5 & 10 & 7.5 \\
\hline \multicolumn{2}{|l|}{ Pain } & 1 & 10 & 1 & 4 & 1 & 1 \\
\hline \multirow[t]{2}{*}{ Hand Function } & 9-HPT (seconds) & 20 & 20 & 18 & 19 & 16 & 18 \\
\hline & Box andBlocks & 24 & 30 & 35 & 31 & 43 & 38 \\
\hline \multirow[t]{2}{*}{ Productive work } & Holding hammer $1 \mathrm{Kg}$ & Able & Unable & Able & Unable & Able & Able \\
\hline & $\begin{array}{c}\text { Using hammer } 1 \mathrm{Kg} 10 \\
\text { knocks }\end{array}$ & Able & Unable & Able & Unable & Able & Able \\
\hline
\end{tabular}

Table 1: Outcome measures with scores of three intakes.

Our case study had modified Kessler repair which is the most popular method of tendon repair. It exemplifies high-strength, low-friction repairs that minimize friction between the tendon and flexor sheath while maintaining sufficient strength to the repair [4]. In regard to the timing of repair, acute tendon injuries re- quire urgent repair within 24 hours of injury. A primary repair can be done within 24 hours and considered delayed primary repair with the 1 st day up to the $14^{\text {th }}$ day [4]. Our case study had delayed primary repair after three days of injury; however, he showed significant improvement in all aspects of management. 
The findings of this case study agree with Griffin., et al. [2] who pointed out that hand therapy after hand tendon repair aims to achieving function and gliding but to avoiding tendon rupture. Method of mobilization is vital for achieving hand therapy goals as well as preventing complications. Griffin mentioned three methods of mobilization, namely: active extension with rubber band flexion; passive motion; and combination of passive and active motion. Despite the debate, mobilized tendons have shown faster healing, stronger healed tendons, and fewer adhesions than immobilization. In our case study, we used active and passive motion approach without a rubber band which was effective.

Early mobilization is associated with better tendon repair outcomes. Tendons have two types of healing, extrinsic and intrinsic. Extrinsic healing is enhanced by postoperative immobilization where fibroblasts migrate from the sheath into the injured site. This explains why immobilization protocols to restore tendon is associated with scar formation at the repair site that limits tendon movements. Intrinsic healing occurs with mobilization of the tendon where tendon cells can migrate across closely approximated ends and heal with nutrients from synovial fluid. Peripheral adhesions do not participate in intrinsic tendon healing [4]. The range of motion in our case study improved; however, the DIP joint was restricted due to possible adhesions formation. Mobilization was done by manual active and passive ranging. Rubber band traction was not used to allow full finger extension without any limitation. Abdelfattah., et al. [4] pointed out that extension loss is a frequent complication when a rubber band is used to improve finger flexion. Abdelfattah added that active and passive motion improves repaired tendon gliding which was followed in our case study.

Our case study valued occupations where productive work was an important motivating factor as he reported in the assessment. Chan and Spencer [5,6] mentioned that occupations have the potential to create a better life for patients after tendon laceration and repair.

\section{Recommendations}

Tendon repair should be performed within the first 24-hours unless other significant findings intervene. Early mobilization using active and passive motion is recommended with precautions to avoid tendon rupture. Patient education to prevent complications, for example, decreased sensation. Occupation enhances patients with tendon repair to involve actively in hand therapy plan.

\section{Conclusion}

The results of flexor tendon repair in the hand have improved which is the result of a combination of improved surgical techniques and better rehabilitation approaches. The gliding of the tendon and hand functions can be achieved better with early mobilization of the repaired finger flexor tendon. Early active mobilisation could reduce the time of rehabilitation and the need for therapists which are current core goals of health management.

\section{Funding}

This research received no specific grant support from any funding agency in the public, commercial, or not-for-profit sectors.

\section{Availability of Data and Materials}

Not Applicable.

\section{Disclosures}

The author did the conception, design, coordination, drafting, reading, and approval of the final manuscript.

\section{Contributor Ship}

Not Applicable.

\section{Ethical Approval}

All procedures followed were in accordance with the ethical standards of the responsible committee on human experimentation (institutional and national) and with the Helsinki Declaration of 1975, as revised in 2000 (5). Informed consent was obtained from the patient for being included in the study. The study was approved by Hamad Medical Director as well. All documents re available with the author for any review.

\section{Informed Consent for Publication}

A written informed consent was obtained from the patient for publication of this case report and any accompanying images. A copy of the written permission is available for review by the Editorin-Chief of this journal.

\section{Declaration of Conflicting Interests}

The authors confirm that there is no conflict of interest with respect to the research, authorship, and/or publication of this article. 


\section{Bibliography}

1. Starr HM., et al. "Flexor Tendon Repair Rehabilitation Protocols: A Systematic Review". The Journal of Hand Surgery 38.9 (2013): 128-139.

2. Griffin M., et al. "An Overview of the Management of Flexor Tendon Injuries". The Open Orthopaedics Journal 6.1 (2012): 28-35.

3. Yang G., et al. "Tendon and ligament regeneration and repair: Clinical relevance and developmental paradigm". Birth Defects Research Part C: Embryo Today: Reviews 99.3 (2013): 203-222.

4. Abdelfattah A., et al. "Flexor Tendon Injuries: A Protocol Based on Factors that Enhance Intrinsic Tendon Healing and Improves the Postoperative Outcome". Egyptian Journal of Plastic and Reconstructive Surgery 33.2 (2009) 145-150.

5. Chan J and Spencer J. "Adaptation to hand injury: An evolving experience". American Journal of Occupational (2004).

6. Chesney A., et al. "Systematic Review of Flexor Tendon Rehabilitation Protocols in Zone II of the Hand". Plastic and Reconstructive Surgery 127.4 (2011): 1583-1592.

\section{Assets from publication with us}

- Prompt Acknowledgement after receiving the article

- Thorough Double blinded peer review

- Rapid Publication

- Issue of Publication Certificate

- High visibility of your Published work

Website: https://www.actascientific.com/

Submit Article: https://www.actascientific.com/submission.php

Email us: editor@actascientific.com

Contact us: +919182824667 